\title{
Displacement of non-Newtonian compressible fluids in plane porous media flow
}

\author{
R. Ugarelli ${ }^{1}$, M. Bottarelli ${ }^{2}$ \& V. Di Federico ${ }^{1}$ \\ ${ }^{I}$ Dip. di Ingegneria delle Strutture, dei Trasporti, delle Acque, \\ del Rilevamento, del Territorio (D.I.S.T.A.R.T.), \\ Università di Bologna, Italy \\ ${ }^{2}$ Dip. di Architettura, Università di Ferrara, Italy
}

\begin{abstract}
Displacement of non-Newtonian fluid in porous media is of paramount importance in the flow modeling of oil reservoirs. Although numerical solutions are available, there exists a need for closed-form solutions in simple geometries. Here we revisit and expand the work of Pascal and Pascal [4], who analyzed the dynamics of a moving stable interface in a semi-infinite porous domain saturated by two fluids, displacing and displaced, both non-Newtonian of power-law behavior, assuming continuity of pressure and velocity at the interface, and constant initial pressure. The flow law for both fluids is a modified Darcy's law. Coupling the nonlinear flow law with the continuity equation considering the fluids compressibility, yields a set of nonlinear second-order PDEs. If the fluids have the same consistency index $n$, the equations can be transformed via a selfsimilar variable; incorporation of the conditions at the interface shows the existence of a compression front ahead of the moving interface. After some algebra, one obtains a set of nonlinear equations, whose solution yields the location of the moving interface and compression front, and the pressure distributions. The previous equations include integrals which can be expressed by analytical functions if $n$ is of the form $k /(k+1)$ or $(2 k-1) /(2 k+1)$, with $k$ a positive integer. Explicit expressions are provided for $k=1,2$; for other values, results are easily obtained via recursive formulae. All results are presented in dimensionless form; the pressure distribution and interface positions are studied and discussed as a function of the self-similar variable for different values of the mobility and compressibility ratios.
\end{abstract}

Keywords: porous media, non-Newtonian, compressible fluids, self-similar solution, immiscible displacement. 


\section{Introduction}

Displacement of non-Newtonian fluid in porous media is of paramount importance in the flow modeling of oil reservoirs, where often a power-law fluid of pseudo-plastic behavior (water thickened with polymer addictive) is used to minimize the instability effects [5]. A large bibliography exists on porous media flow of non-Newtonian fluids: see for example the papers by $\mathrm{H}$. Pascal and coworkers and references therein [2,3]. Although numerical solutions are available [5], there exists a need for closed-form solutions in simple geometries. Pascal and Pascal [4] analyzed the dynamics of a moving stable interface in a semi-infinite porous domain saturated by two fluids, displacing and displaced, both non-Newtonian of power-law behavior. In this paper, we revisit and expand their work by providing: i) a dimensionless formulation of the problem; ii) explicit expressions for the variables of interest for special values of the flow law exponent; iii) a discussion of their behavior as functions of the problem parameters.

\section{Problem formulation}

We analyze the dynamics of a moving interface due to fluid injection of a nonNewtonian fluid in a semi-infinite porous domain of constant thickness $m$ saturated by another non-Newtonian fluid (Figure 1); both fluids, displacing and displaced, are of power-law pseudo-plastic behavior with the same consistency index $n<1$. The interface is stable, so that a piston-like displacement exists; the pressure and velocity fields are assumed to be continuous at the interface; the pressure is taken to be constant and equal to $p_{w}$ in the domain occupied by the displaced fluid at time $t=0$; the displacing fluid is injected at a constant pressure $p_{e}$ greater than the ambient pressure $p_{w}$.

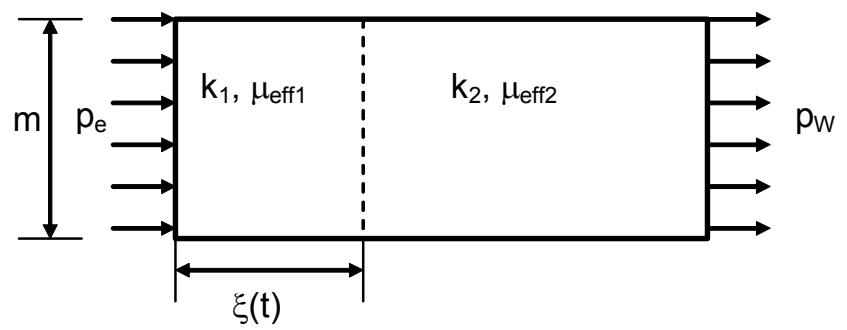

Figure 1: Definition sketch.

The flow law for both fluids is a modified Darcy's law taking into account the nonlinearity of the rheological equation [2-4]. Thus the flow and continuity equation for both fluids ( $i=1$ for the displacing, $i=2$ for the displaced fluid) are:

$$
v_{i}=\left(-\frac{k_{i}}{\mu_{e f i}} \frac{\partial p_{i}}{\partial x}\right)^{1 / n}, \frac{\partial v_{i}}{\partial x}=-c_{0 i} \phi \frac{\partial p_{i}}{\partial t},
$$


where $x$ denotes the spatial coordinate, $t$ time, $v$ Darcy velocity, $p$ pressure, $n$ fluid consistency index, $c_{0}$ compressibility coefficient, $\phi$ porosity, $k$ permeability coefficient, $\mu_{e f}$ effective viscosity; in turn, the ratio between the latter two is a function of $n, \phi, k$ (see for example eqn 4 in [4]). Substituting eqn (1b) in eqn (1a) one obtains for the two fluids $(i=1,2)$ :

$$
\frac{\partial^{2} p_{i}}{\partial x^{2}}=n c_{0 i} \phi\left(\frac{\mu_{e f i}}{k_{i}}\right)^{1 / n}\left(\frac{\partial p_{i}}{\partial x}\right)^{(n-1) / n} \frac{\partial p_{i}}{\partial t},
$$

where $p_{i}(x, t)=p_{1}(x, t)$ for $0 \leq x \leq \xi(t)$ and $p_{i}(x, t)=p_{2}(x, t)$ for $\xi(t) \leq x<\infty$, with $\xi(t)$ interface position and $\xi(t=0)=0$. The injection flowrate per unit width is $q(t)=v_{1}(0, t) m$. Initial and boundary conditions are:

$$
p_{1}(x, 0)=p_{e} ; p_{1}(0, t)=p_{e} ; \lim _{x \rightarrow \infty} p_{2}(x, t)=p_{w} .
$$

At the moving interface, the pressure and velocity fields are continuous; thus

$$
p_{1}[\xi(t), t]=p_{2}[\xi(t), t] ;\left(-\frac{k_{1}}{\mu_{e f 1}} \frac{\partial p_{1}}{\partial x}\right)_{x=\xi(t)}^{1 / n}=\left(-\frac{k_{2}}{\mu_{e f 2}} \frac{\partial p_{1}}{\partial x}\right)_{x=\xi(t)}^{1 / n}=V,
$$

in which $V=\phi d \xi / d t$ is the common value of the Darcy velocity at the interface. We now define the following dimensionless variables $(i=1,2)$ :

$$
\left(x^{\prime}, \xi^{\prime}, t^{\prime}, p_{i}^{\prime}, v_{i}^{\prime}, V^{\prime}, q^{\prime}\right)=\left(\frac{x}{m}, \frac{\xi}{m}, \frac{t}{T}, c_{0 i} p_{i}, \frac{v_{i} T}{\phi m}, \frac{V T}{\phi m}, \frac{q T}{\phi m^{2}}\right),
$$

where $T=c_{01}^{1 / n}\left(\mu_{e f 1} / k_{1}\right)^{1 / n} \phi m^{(n+1) / n}$ is a timescale. This recasts eqns (1a) and (2), respectively, in the following dimensionless forms, where primes are dropped for convenience:

$$
\begin{gathered}
v_{1}=\left(-\frac{\partial p_{1}}{\partial x}\right)^{1 / n} ; v_{2}=\left(-M \frac{\partial p_{2}}{\partial x}\right)^{1 / n}, \\
\frac{\partial^{2} p_{1}}{\partial x^{2}}=n\left(\frac{\partial p_{1}}{\partial x}\right)^{(n-1) / n} \frac{\partial p_{1}}{\partial t} ; \frac{\partial^{2} p_{2}}{\partial x^{2}}=n \frac{\alpha}{M^{1 / n}}\left(\frac{\partial p_{2}}{\partial x}\right)^{(n-1) / n} \frac{\partial p_{2}}{\partial t},
\end{gathered}
$$

in which $M=\left(k_{2} / \mu_{e f 2}\right) /\left(k_{1} / \mu_{e f 1}\right)$ and $\alpha=c_{02} / c_{01}$ are respectively the mobility ratio and the compressibility ratio. Conditions at the interface (4a)-(4b) read in dimensionless form (primes omitted)

$$
p_{1}[\xi(t), t]=p_{2}[\xi(t), t] ;\left(-\frac{\partial p_{1}}{\partial x}\right)_{x=\xi(t)}^{1 / n}=\left(-M \frac{\partial p_{2}}{\partial x}\right)_{x=\xi(t)}^{1 / n}=V=\frac{d \xi}{d t},
$$

while initial and boundary conditions (3a)-(3c) remain unchanged. 


\section{Solution of the problem}

Introducing the similarity variable

$$
\eta=\frac{x}{t^{n /(n+1)}}
$$

eqns (6a)-(6b) and (7a)-(7b) take the form

$$
\begin{gathered}
v_{1}=t^{-1 / n+1}\left(-\frac{d p_{1}}{d \eta}\right)^{1 / n}, v_{2}=t^{-1 / n+1}\left(-\frac{1}{M} \frac{d p_{2}}{d \eta}\right)^{1 / n}, \\
\frac{d^{2} p_{1}}{d \eta^{2}}-\frac{n^{2}}{n+1} \eta\left(-\frac{d p_{1}}{d \eta}\right)^{(2 n-1) / n}=0,\left(0 \leq \eta \leq \eta_{1}\right), \\
\frac{d^{2} p_{2}}{d \eta^{2}}-\frac{n^{2}}{n+1} \frac{\alpha}{M^{1 / n}} \eta\left(-\frac{d p_{2}}{d \eta}\right)^{(2 n-1) / n}=0,\left(\eta_{1} \leq \eta<\infty\right),
\end{gathered}
$$

where $\eta_{1}$ is linked to the position of the moving interface by

$$
\xi(t)=\eta_{1} t^{n /(n+1)}
$$

Initial and boundary conditions ( $3 a)-(3 c)$ coalesce into

$$
p_{1}(0)=p_{e}, \lim _{\eta \rightarrow \infty} p_{2}(\eta)=p_{w},
$$

while the conditions at the interface $(8 \mathrm{a})-(8 \mathrm{~b})$ become

$$
p_{1}\left(\eta_{1}\right)=p_{2}\left(\eta_{1}\right)=p_{\text {int }} ;\left(\frac{d p_{1}}{d \eta}\right)_{\eta=\eta_{1}}=M\left(\frac{d p_{2}}{d \eta}\right)_{\eta=\eta_{1}},
$$

in which $p_{\text {int }}$ is the pressure at the interface. The interface velocity becomes

$$
V=\frac{d \xi}{d t}=t^{-1 /(n+1)}\left(-\frac{d p_{1}}{d \eta}\right)_{\eta=\eta_{1}}^{1 / n} .
$$

Integrating eqn (16) with the initial condition $\xi(t=0)=0$ yields

$$
\xi(t)=\frac{n+1}{n} t^{n /(n+1)}\left(-\frac{d p_{1}}{d \eta}\right)_{\eta=\eta_{1}}^{1 / n} .
$$

Coupling eqns (13) and (17) leads to the following expression for $\eta_{1}$

$$
\eta_{1}=\xi(t) t^{-n /(n+1)}=\frac{1+n}{n}\left(-\frac{d p_{1}}{d \eta}\right)_{\eta=\eta_{1}}^{1 / n} .
$$

Now integrating once eqns (11)-(12) yields respectively 


$$
\begin{gathered}
\frac{d p_{1}}{d \eta}=-\left[\left(-\frac{d p_{1}}{d \eta}\right)_{\eta=\eta_{1}}^{(1-n) / n}-\frac{n(1-n)}{2(1+n)}\left(\eta^{2}-\eta_{1}^{2}\right)\right]^{n /(1-n)} \quad\left(0 \leq \eta \leq \eta_{1}\right), \\
\frac{d p_{2}}{d \eta}=-\left[\left(-\frac{d p_{2}}{d \eta}\right)_{\eta=\eta_{1}}^{(1-n) / n}-\frac{n(1-n) \alpha}{2(1+n) M^{1 / n}}\left(\eta^{2}-\eta_{1}^{2}\right)\right]^{n /(1-n)} \quad\left(\eta_{1} \leq \eta<\infty\right) .
\end{gathered}
$$

Note that eqn (19) is in variance with (18) of Pascal and Pascal [4]. From eqn (20) it is evident that $d p_{2} / d \eta=0$ for

$$
\eta^{*}=\left[\eta_{1}^{2}+\frac{2(1+n) M^{1 / n}}{n(1-n) \alpha}\left(-\frac{d p_{2}}{d \eta}\right)_{\eta=\eta_{1}}^{(1-n) / n}\right]^{1 / 2} .
$$

Eqn (21) shows the existence of a compression front ahead of the moving interface, whose dimensionless position and velocity are defined by

$$
\xi *(t)=\eta^{*} t^{n /(n+1)} ; V^{*}=\frac{d \xi *}{d t}=\frac{n}{n+1} \eta^{*} t^{-1 /(n+1)} .
$$

At and beyond the compression front, the displaced fluid Darcy velocity $v_{2}$ is null; hence, the fluid remains at the constant pressure $p_{w}$ for $\eta \geq \eta *$. Therefore, the boundary condition (14b) is replaced by $p_{2}\left(\eta^{*}\right)=p_{w}$; taking this and eqn (14a) into account, the integration of eqns (19)-(20) yields

$$
\begin{aligned}
& p_{1}(\eta)=p_{e}-\int_{0}^{\eta}\left[\left(-\frac{d p_{1}}{d \eta}\right)_{\eta=\eta_{1}}^{(1-n) / n}-\frac{n(1-n)}{2(1+n)}\left(\tau^{2}-\eta_{1}^{2}\right)\right]^{n /(1-n)} d \tau, \\
& p_{2}(\eta)=p_{w}+\int_{\eta}^{\eta^{*}}\left[\left(-\frac{d p_{2}}{d \eta}\right)_{\eta=\eta_{1}}^{(1-n) / n}-\frac{n(1-n) \alpha}{2(1+n) M^{1 / n}}\left(\tau^{2}-\eta_{1}^{2}\right)\right]^{n /(1-n)} d \tau .
\end{aligned}
$$

On the other hand, subtracting eqn (24) from eqn (23) and taking into account the interface condition (15a) gives

$$
\begin{aligned}
& \Delta p=p_{e}-p_{w}=\int_{0}^{\eta_{1}}\left[\left(-\frac{d p_{1}}{d \eta}\right)_{\eta=\eta_{1}}^{(1-n) / n}-\frac{n(1-n)}{2(1+n)}\left(\tau^{2}-\eta_{1}^{2}\right)\right]^{n /(1-n)} d \tau+ \\
& +\int_{\eta_{1}}^{\eta^{*}}\left[\left(-\frac{d p_{2}}{d \eta}\right)_{\eta=\eta_{1}}^{(1-n) / n}-\frac{n(1-n) \alpha}{2(1+n) M^{1 / n}}\left(\tau^{2}-\eta_{1}^{2}\right)\right]^{n /(1-n)} d \tau
\end{aligned}
$$

The nonlinear algebraic system of equations (15b), (18), (21), and (25), for assigned pressure drop $\Delta p$, compressibility ratio $\alpha$, and mobility ratio $M$, determines the pressure gradients at the interface, and the positions of the moving interface and of the compression fronts, i.e. $\left(d p_{1} / d \eta\right)_{\eta=\eta_{1}},\left(d p_{2} / d \eta\right)_{\eta=\eta_{1}}$, 
$\eta_{1}, \eta^{*}$; once these are known, the pressure distributions are easily determined. To solve the set of equations, we proceed as follows: first, we rewrite eqn (21), with the aid of eqs (15b) and (18), as

$$
\eta^{*}=\eta_{1}\left[1+\frac{2(1+n)^{n} M}{n^{n}(1-n) \alpha \eta_{1}^{1+n}}\right]^{1 / 2}=\eta_{1} c_{1}\left(\eta_{1}\right) .
$$

Second, with the help of eqns (15b), (18), and (26), eqn (25) becomes

$$
\begin{aligned}
& \Delta p=\int_{0}^{\eta_{1}}\left[\left(\frac{n}{1+n} \eta_{1}\right)^{1-n}-\frac{n(1-n)}{2(1+n)}\left(\tau^{2}-\eta_{1}^{2}\right)\right]^{n /(1-n)} d \tau+ \\
& +\int_{\eta_{1}}^{\eta_{1} c_{1}\left(\eta_{1}\right)}\left[\frac{1}{M^{(1-n) / n}}\left(\frac{n}{1+n} \eta_{1}\right)^{1-n}-\frac{n(1-n) \alpha}{2(1+n) M^{1 / n}}\left(\tau^{2}-\eta_{1}^{2}\right)\right]^{n /(1-n)} d \tau
\end{aligned}
$$

in which the only unknown is $\eta_{1}$; once $\eta_{1}$ is determined via eqn (27), $\eta^{*}$ is then calculated through the function $c_{1}\left(\eta_{1}\right)$ defined in eqn (26). Finally, the pressure distributions behind and ahead the moving interface are given by eqns (23)-(24), which become, with the aid of eqns (15b), (18), and (21)

$$
\begin{gathered}
p_{1}(\eta)=p_{e}-\int_{0}^{\eta}\left[\left(\frac{n}{1+n} \eta_{1}\right)^{1-n}-\frac{n(1-n)}{2(1+n)}\left(\tau^{2}-\eta_{1}^{2}\right)\right]^{n /(1-n)} d \tau, \\
p_{2}(\eta)=p_{w}+\int_{\eta}^{\eta_{1} c_{1}\left(\eta_{1}\right)}\left[\frac{1}{M^{(1-n) / n}}\left(\frac{n}{1+n} \eta_{1}\right)^{1-n}-\frac{n(1-n) \alpha}{2(1+n) M^{1 / n}}\left(\tau^{2}-\eta_{1}^{2}\right)\right]^{n /(1-n)} d \tau .
\end{gathered}
$$

The integrals in eqns (27)-(29) are of the type $\int\left(a-b \tau^{2}\right)^{p} d \tau$, with $a=a\left(\eta_{1}\right)$ and $b$ positive quantities, and $p$ a positive real number: hence, they can be expressed as analytical functions in two cases [1, p. 84]: i) $n=k /(1+k)$; ii) $n=(2 k-1) /(2 k+1)$, where $k$ is a positive integer.

\section{Closed-form results}

In the following, explicit expressions for all variables of interest are provided for cases i) and ii) for $k=1$ and $k=2$, while for other values of $k$ results are easily obtained via recursive formulae [1]; for each case, we report eqns (26)-(29), the expressions of the coefficients included therein, the position and velocity of the interface and of the compression front, and the dimensionless injection flowrate per unit width $q(t)$; for the sake of brevity, $c_{1}\left(\eta_{1}\right)=c_{1}, a_{1}\left(\eta_{1}\right)=a_{1}, a_{2}\left(\eta_{1}\right)=a_{2}$.

4.1 Case i) $n=k /(k+1) ; k=1(n=1 / 2)$

$$
c_{1}=\left[1+\frac{4(3)^{1 / 2} M}{\alpha \eta_{1}^{3 / 2}}\right]^{1 / 2},
$$




$$
\begin{gathered}
\Delta p=\eta_{1}\left[a_{1}+\left(c_{1}-1\right) a_{2}\right]-\frac{\eta_{1}^{3}}{3}\left[b_{1}+\left(c_{1}^{3}-1\right) b_{2}\right] \\
p_{1}(\eta)=p_{e}-a_{1} \eta+\frac{b_{1} \eta^{3}}{3}, p_{2}(\eta)=p_{w}+a_{2}\left(c_{1} \eta_{1}-\eta\right)-\frac{b_{2}}{3}\left(c_{1}^{3} \eta_{1}^{3}-\eta^{3}\right), \\
a_{1}=\left(\frac{\eta_{1}}{3}\right)^{1 / 2}+\frac{\eta_{1}^{2}}{12}, b_{1}=\frac{1}{12}, a_{2}=\frac{1}{M}\left(\frac{\eta_{1}}{3}\right)^{1 / 2}+\frac{\alpha \eta_{1}^{2}}{12 M^{2}}, b_{2}=\frac{\alpha}{12 M^{2}}, \\
\xi(t)=\eta_{1} t^{1 / 3}, V(t)=\frac{\eta_{1}}{3} t^{-2 / 3}, \xi *(t)=c_{1} \eta_{1} t^{1 / 3}, V^{*}(t)=\frac{c_{1} \eta_{1}}{3} t^{-2 / 3}, \\
q(t)=a_{1}^{2} t^{-2 / 3} .
\end{gathered}
$$

4.2 Case i) $n=k /(k+1) ; k=2(n=2 / 3)$

$$
\begin{gathered}
c_{1}=\left[1+\frac{3(50)^{1 / 3} M}{\alpha \eta_{1}^{5 / 3}}\right]^{1 / 2} \\
\Delta p=\eta_{1}\left[a_{1}^{2}+\left(c_{1}-1\right) a_{2}^{2}\right]-\frac{2 \eta_{1}^{3}}{3}\left[a_{1} b_{1}+\left(c_{1}^{3}-1\right) a_{2} b_{2}\right]+\frac{\eta_{1}^{5}}{5}\left[b_{1}^{2}+\left(c_{1}^{5}-1\right) b_{2}^{2}\right] \\
p_{1}(\eta)=p_{e}-a_{1} \eta+\frac{2}{3} a_{1} b_{1} \eta^{3}-\frac{1}{5} b_{1}^{2} \eta^{5} \\
p_{2}(\eta)=p_{w}+a_{2}^{2}\left(c_{1} \eta_{1}-\eta\right)-\frac{2}{3} a_{2} b_{2}\left(c_{1}^{3} \eta_{1}^{3}-\eta^{3}\right)+\frac{1}{5} b_{2}^{2}\left(c_{1}^{5} \eta_{1}^{5}-\eta^{5}\right) \\
a_{1}=\left(\frac{2 \eta_{1}}{5}\right)^{1 / 3}+\frac{\eta_{1}^{2}}{15}, b_{1}=\frac{1}{15}, \\
a_{2}=\frac{1}{M^{1 / 2}}\left(\frac{2 \eta_{1}}{5}\right)^{1 / 3}+\frac{\alpha \eta_{1}^{2}}{15 M^{3 / 2}}, b_{2}=\frac{\alpha}{15 M^{3 / 2}} \\
\qquad(t)=\eta_{1} t^{2 / 5}, V(t)=\frac{2 \eta_{1}}{5} t^{-3 / 5}, \xi *(t)=c_{1} \eta_{1} t^{2 / 5}, V^{*}(t)=\frac{2 c_{1} \eta_{1}}{5} t^{-3 / 5} \\
q(t)=a_{1}^{3} t^{-3 / 5} .
\end{gathered}
$$

4.3 Case ii) $n=(2 k-1) /(2 k+1) ; k=1(n=1 / 3)$

$$
\begin{gathered}
c_{1}=\left[1+\frac{3(4)^{1 / 3} M}{\alpha \eta_{1}^{4 / 3}}\right]^{1 / 2}, \\
\Delta p=\frac{\eta_{1}}{2}\left[\left(a_{1}-b_{1} \eta_{1}^{2}\right)^{1 / 2}+c_{1}\left(a_{2}-b_{2} c_{1}^{2} \eta_{1}^{2}\right)^{1 / 2}-\left(a_{2}-b_{2} \eta_{1}^{2}\right)^{1 / 2}\right]+ \\
+\frac{a_{1}}{2 b_{1}^{1 / 2}} \arcsin \left[\left(\frac{b_{1}}{a_{1}}\right)^{1 / 2} \eta_{1}\right]+\frac{a_{2}}{2 b_{2}^{1 / 2}}\left\{\arcsin \left[\left(\frac{b_{2}}{a_{2}}\right)^{1 / 2} c_{1} \eta_{1}\right]-\arcsin \left[\left(\frac{b_{2}}{a_{2}}\right)^{1 / 2} \eta_{1}\right]\right\}
\end{gathered}
$$




$$
\begin{gathered}
p_{1}(\eta)=p_{e}-\frac{\eta}{2}\left(a_{1}-b_{1} \eta^{2}\right)^{1 / 2}-\frac{a_{1}}{2 b_{1}^{1 / 2}} \arcsin \left[\left(\frac{b_{1}}{a_{1}}\right)^{1 / 2} \eta\right], \\
p_{2}(\eta)=p_{w}+\frac{1}{2}\left[c_{1} \eta_{1}\left(a_{2}-b_{2} c_{1}^{2} \eta_{1}^{2}\right)^{1 / 2}-\eta\left(a_{2}-b_{2} \eta^{2}\right)^{1 / 2}\right]+ \\
+\frac{a_{2}}{2 b_{2}^{1 / 2}}\left\{\arcsin \left[\left(\frac{b_{2}}{a_{2}}\right)^{1 / 2} c_{1} \eta_{1}\right]-\arcsin \left[\left(\frac{b_{2}}{a_{2}}\right)^{1 / 2} \eta\right]\right\} \\
a_{1}=\left(\frac{\eta_{1}}{4}\right)^{2 / 3}+\frac{\eta_{1}^{2}}{12}, b_{1}=\frac{1}{12}, a_{2}=\frac{1}{M^{2}}\left(\frac{\eta_{1}}{4}\right)^{2 / 3}+\frac{\alpha \eta_{1}^{2}}{12 M^{3}}, b_{2}=\frac{\alpha}{12 M^{3}},(48 \mathrm{a}, \mathrm{b}, \mathrm{c}, \mathrm{d}) \\
\xi(t)=\eta_{1} t^{1 / 4}, V(t)=\frac{\eta_{1}}{4} t^{-3 / 4}, \xi^{*}(t)=c_{1} \eta_{1} t^{2 / 5}, V^{*}(t)=\frac{c_{1} \eta_{1}}{4} t^{-3 / 5}, \quad(49 \mathrm{a}, \mathrm{b}, \mathrm{c}, \mathrm{d}) \\
q(t)=a_{1}^{3 / 2} t^{-3 / 4} .
\end{gathered}
$$

\subsection{Case ii) $n=(2 k-1) /(2 k+1) ; k=2(n=3 / 5)$}

$$
c_{1}=\left[1+5\left(\frac{8}{3}\right)^{3 / 5} \frac{M}{\alpha \eta_{1}^{8 / 5}}\right]^{1 / 2},
$$

$$
\Delta p=\frac{\eta_{1}}{4}\left[\left(a_{1}-b_{1} \eta_{1}^{2}\right)^{3 / 2}+c_{1}\left(a_{2}-b_{2} c_{1}^{2} \eta_{1}^{2}\right)^{3 / 2}-\left(a_{2}-b_{2} \eta_{1}^{2}\right)^{3 / 2}\right]+
$$$$
\frac{3 \eta_{1}}{8}\left[a_{1}\left(a_{1}-b_{1} \eta_{1}^{2}\right)^{1 / 2}+a_{2} c_{1}\left(a_{2}-b_{2} c_{1}^{2} \eta_{1}^{2}\right)^{1 / 2}-a_{2}\left(a_{2}-b_{2} \eta_{1}^{2}\right)^{1 / 2}\right]+
$$

$+\frac{3 a_{1}^{2}}{8 b_{1}^{1 / 2}} \arcsin \left[\left(\frac{b_{1}}{a_{1}}\right)^{1 / 2} \eta_{1}\right]+\frac{3 a_{2}^{2}}{8 b_{2}^{1 / 2}}\left\{\arcsin \left[\left(\frac{b_{2}}{a_{2}}\right)^{1 / 2} c_{1} \eta_{1}\right]-\arcsin \left[\left(\frac{b_{2}}{a_{2}}\right)^{1 / 2} \eta_{1}\right]\right\}$

$$
p_{1}(\eta)=p_{e}-\frac{1}{4}\left(a_{1}-b_{1} \eta^{2}\right)^{3 / 2}-\frac{3 a_{1} \eta}{8}\left(a_{1}-b_{1} \eta^{2}\right)^{1 / 2}-\frac{3 a_{1}^{2}}{8 b_{1}^{1 / 2}} \arcsin \left[\left(\frac{b_{1}}{a_{1}}\right)^{1 / 2} \eta\right],
$$

$$
\begin{aligned}
& p_{2}(\eta)=p_{w}+\frac{1}{4}\left[c_{1} \eta_{1}\left(a_{2}-b_{2} c_{1}^{2} \eta_{1}^{2}\right)^{3 / 2}-\eta\left(a_{2}-b_{2} \eta^{2}\right)^{3 / 2}\right]+ \\
& +\frac{3 a_{2}}{8}\left[c_{1} \eta_{1}\left(a_{2}-b_{2} c_{1}^{2} \eta_{1}^{2}\right)^{1 / 2}-\eta\left(a_{2}-b_{2} \eta^{2}\right)^{1 / 2}\right]+ \\
& +\frac{3 a_{2}^{2}}{8 b_{2}^{1 / 2}}\left\{\arcsin \left[\left(\frac{b_{2}}{a_{2}}\right)^{1 / 2} c_{1} \eta_{1}\right]-\arcsin \left[\left(\frac{b_{2}}{a_{2}}\right)^{1 / 2} \eta\right]\right\}
\end{aligned}
$$




$$
\begin{gathered}
a_{1}=\left(\frac{3 \eta_{1}}{8}\right)^{2 / 5}+\frac{3 \eta_{1}^{2}}{40}, b_{1}=\frac{3}{40}, \\
a_{2}=\frac{1}{M^{2 / 3}}\left(\frac{3 \eta_{1}}{8}\right)^{2 / 5}+\frac{3 \alpha \eta_{1}^{2}}{40 M^{5 / 3}}, b_{2}=\frac{3 \alpha}{40 M^{5 / 3}}, \\
\xi(t)=\eta_{1} t^{3 / 8}, V(t)=\frac{3 \eta_{1}}{8} t^{-5 / 8}, \begin{array}{c}
\xi *(t)=c_{1} \eta_{1} t^{3 / 8}, V^{*}(t)=\frac{3 c_{1} \eta_{1}}{8} t^{-5 / 8} \\
q(t)=a_{1}^{5 / 2} t^{-5 / 8}
\end{array}
\end{gathered}
$$

\section{Discussion of results}

In this section we discuss the behavior of the variables of interest as functions of the fluid properties. We do so for the reference case $p_{e}=1, p_{w}=0.5$; hence $\Delta p=0.5$. We evaluate results for $n=0.33,0.50,0.60,0.67$; these values cover quite well the range of variation of $n$ for pseudoplastic fluids in field cases. Finally, to grasp the influence of fluid mobility and compressibility, we consider the following cases for the mobility ratio $M$ and compressibility ratio $\alpha$ : I) $M=$ $0.2, \alpha=0.2$; II) $M=0.2, \alpha=5$; III) $M=5, \alpha=0.2$; IV) $M=5, \alpha=5$. In Figures 2a-d, the interface location $\xi(t)$ is illustrated as a function of time for the above values of $n, M$, and $\alpha$.

When the displaced fluid is less compressible and less mobile than the displacing one (case I), the interface advances very slowly, more so for lesser values of $n$ (Figure 2a); when the displaced fluid is more compressible and more mobile than the displacing one (case IV), the interface advances very fast, more so for larger values of $n$, even if the difference between results for different values of $n$ is, relatively speaking, less marked than in the previous case (Figure 2d). When the displaced fluid is less mobile, but more compressible than the displacing one (case II), the interface advances almost as fast as for case I, and differences between results for different values of $n$ are as marked as for case IV. Finally, when the displaced fluid is more mobile, but less compressible than the displacing one (case III), the interface advances faster than for case I, but less fast than for cases II and IV; this indicates, at least for the range of values of parameters examined here, that to achieve the maximum displacement, one needs not only large values of the power law model exponent $n$, but also of the compressibility and mobility ratio, the former more than the latter.

Figure 3 a shows the compression front location $\xi *(t)$ as a function of time in the four cases listed above for $n=0.50$. As expected, the compression front advances fastest when the displaced fluid is more mobile, but less compressible than the displacing one (case III); the compression front is slower in cases I and IV, and becomes slowest for case II, when the displaced fluid is less mobile and more compressible than the displacing one.

On comparing Figure $3 \mathrm{a}$ with Figures $2 \mathrm{a}-\mathrm{d}$, it is noted that the compression front location is farther from the interface location when the displaced fluid is 
less compressible than the displacing one (cases I and III); the reverse is true when the displaced fluid is more compressible than the displacing one (cases II and IV). The above conclusions hold true also for different values of $n$ (not shown).
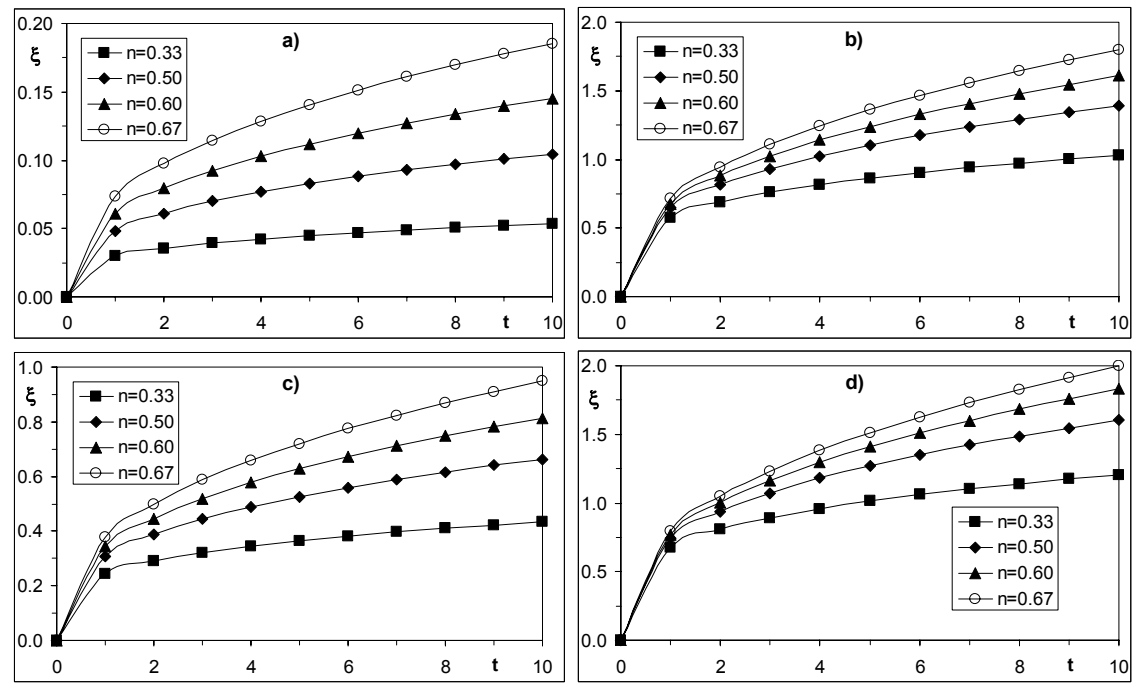

Figure 2: Interface location as a function of time for a) case I: $M=0.2, \alpha=$ 0.2 ; b) case II: $M=0.2, \alpha=5$; c) case III: $M=5, \alpha=0.2$; d) case IV: $M=5, \alpha=5$.
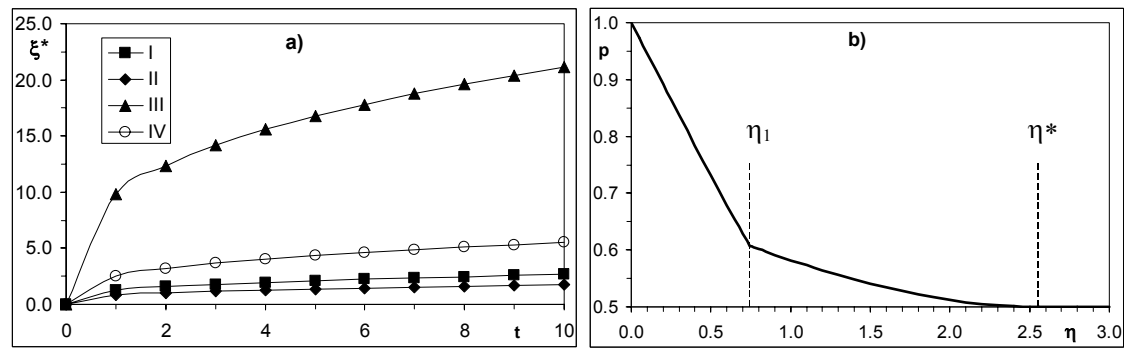

Figure 3: a) Compression front location as a function of time for Cases I-IV and $n=0.50 ; \mathrm{b}$ ) Pressure as a function of $\eta$ for $n=0.50$, Case IV.

Finally, Figure $3 \mathrm{~b}$ shows the behavior of pressure in the displacing and displaced fluids, $p_{1}(\eta)$ and $p_{2}(\eta)$, as a function of $\eta$ for $n=0.50$ and case IV; note the discontinuity in the pressure derivative at the interface location $\eta_{1}=$ 0.745 and the pressure asymptote at the compression front location $\eta^{*}=2.556$. 


\section{Conclusions}

In this paper, we examined the dynamics of non-Newtonian interfaces when a fluid is injected at a constant pressure into a plane porous domain, revisiting and expanding the work of Pascal and Pascal [4]. Via the adoption of a self-similar variable, we obtained closed-form solutions for the pressure distribution, location and velocity of the moving interface and compression front for several values of the power law model exponent.

Expressing the model equations in dimensionless form, it was found that the variables of interest are functions of the imposed pressure difference, of the fluid consistency index (power law model exponent), and of the relative mobility and compressibility of the displacing and displaced fluids.

We confirmed the existence of a pressure front ahead of the moving interface, traveling with finite velocity. Evaluation of the interface location showed that, for a given power law model exponent, a large relative compressibility is comparatively more efficient than a large relative mobility ratio towards maximizing the resulting displacement.

Our results may help in better interpreting transient pressure response in polymer flooding projects in oil reservoir engineering.

\section{References}

[1] Gradshteyn, I.S., \& Ryzhik, I.M., Table of Integrals, Series, and Products, edited by A. Jeffrey, Academic Press: San Diego, 1994.

[2] Pascal, H. \& Pascal F., Flow of non-Newtonian fluid through porous media, Int. J. Engng. Sci., 23(5), pp. 571-585, 1985.

[3] Pascal, H., On the existence of self-similar solutions of the equations governing unsteady flow through a porous medium, Int. J. Heat and Fluid Flow, 9(4), pp. 381-389, 1988.

[4] Pascal, H. \& Pascal F., Displacement of non-Newtonian fluid interfaces in a porous medium: compressible fluid, J. Non-Newt. Fluid Mech., 28, pp. $227-$ 238, 1988.

[5] Wu, Y.-S. \& Pruess, K., A numerical method for simulating non-Newtonian fluid flow and displacement in porous media, Adv. in Wat Res., 21, pp. 351362, 1998. 